DOI: https://doi.org/10.46296/yc.v4i7edesp.0058

\title{
INFLUENCIA DE LA CUARENTENA EN LOS NIVELES DE ANSIEDAD DE LOS CIUDADANOS DE LA PARROQUIA ÁNGEL PEDRO GILER DEL CANTÓN TOSAGUA
}

\section{INFLUENCE OF THE QUARANTINE IN ANXIETY LEVELS OF THE CITIZENS OF THE PARISH ÁNGEL PEDRO GILER OF TOSAGUA CANTON}

\author{
Vélez-Intriago María Milena ${ }^{1 *}$; Navia-Rodríguez Isidro² \\ 1,2 Universidad Técnica de Manabí, UTM. Portoviejo, Ecuador. \\ *Correo: mvelez3907@utm.edu.ec
}

\begin{abstract}
Resumen
La siguiente investigación tiene como finalidad determinar la influencia que ha tenido la cuarentena a causa de la pandemia en el presente año 2020, en los niveles de ansiedad de la población. Siendo el responsable el nuevo coronavirus SARS-CoV-2 (Covid-19), centrándose en el cambio social (aislamiento y distanciamiento social), que se tomó como medida preventiva para el contagio o propagación del virus, el cual nos lleva a la cuarentena y cómo este ha repercutido en la salud mental de las personas. El estudio se realizará con la población de la parroquia "Ángel Pedro Giler", cantón Tosagua correspondiente a la provincia de Manabí, una de las más afectadas en esta provincia por el número de contagios y decesos. El estado mental de las personas estará orientada a los niveles de ansiedad de la población escogida, realizando encuestas y aplicando test de ansiedad (Dass-21 y BECK) para medir el nivel de éste en las personas del presente estudio.
\end{abstract}

Palabras clave: Ansiedad, cuarentena, pandemia.

\begin{abstract}
The following research aims to determine the influence that the quarantine has had due to the pandemic of the current year, 2020, on the population. Being responsible the new coronavirus SARS-CoV-2 (Covid-19), focusing on social change (isolation and social distancing), which was taken as a preventive measure for the spread or contagion of the virus, this leads us to quarantine and how it has affected people's mental health. The study will be carried out with the population of the community "Ángel Pedro Giler", corresponding to the province of Manabí, one of the most affected in Ecuador by numbers of infections and deaths. The mental state of the people will be oriented to the anxiety levels of the chosen population. Carrying out surveys and applying anxiety tests (Dass-21 and BECK) to be able to measure the level of anxiety on the people of the presented study.
\end{abstract}

Keywords: Anxiety, quarantine, pandemic

Información del manuscrito:

Fecha de recepción: 08 de septiembre de 2020.

Fecha de aceptación: 10 de noviembre de 2020.

Fecha de publicación: 16 de noviembre de 2020. 


\section{Introducción}

La propagación del nuevo coronavirus (Covid-19) en más de 180 países, generó una conmoción dentro de la población en un tiempo de estimación corta. Por lo que se lo consideró como una pandemia (Organización Mundial de la Salud (OMS), 2020).

El cambio que se dio en la vida cotidiana de las personas dio como resultado un golpe en el área social y por su puesto en el campo de la salud física y mental. El poco conocimiento que se tenía sobre el Covid-19 ocasionó incertidumbre y temor en las personas, al ser una nueva cepa del coronavirus no se tenía precedentes de este y su afectación en el ser humano. En la actualidad, se ha podido obtener más información de este, según la OMS (2020) puede ser causa de diversas enfermedades humanas, que van desde el resfriado común hasta el SRAS (síndrome respiratorio agudo severo), dichas infecciones suelen cursar con fiebre y síntomas respiratorios. Es así que se clasifica como síntomas principales a la fiebre, tos seca y cansancio. Pero también puede presentar perdida en el sentido del olfato, gusto y ciertos problemas gastrointestinales.

El brote de este virus empezó en diciembre del 2019 en Wuhan, China e ingreso a Ecuador a mediados del mes de febrero, su propagación fue tan veloz que para el mes de abril Ecuador contaba con más de 22.000 casos confirmados. Encontrándose a la provincia de Manabí como una de las zonas más afectadas en número de contagio, contando con más de 8309 casos en el mes de abril (Ministerio de Salud Pública (MSP), 2020).

Con estas cifras, se situó a Ecuador como el segundo lugar de América Latina en contagios (Corporación Británica de Radiodifusión (BBC), 2020), motivo por el cual se tomó como medida preventiva el inicio del aislamiento social (Cuarentena), lo que generó un cambio notable en el estilo de vida a la que estaba acostumbrada la población. Dicho de otra manera, la epidemia actual representa una crisis en la vida de los seres humanos, lo que implica un cambio repentino y significativo de la situación vital del individuo (Rodríguez et al., 2020).

Siendo así, un impacto para la homeostasis de la psique del ser 
humano, viéndose comprometida la salud mental de las personas, puesto que en situaciones de crisis los valores individuales y colectivos son modificados o suprimidos y se asiste a irregulares actuaciones y conductas, esto se convierte en un factor de riesgo para la salud psicológica (Rodríguez et al., 2020)

Dicho eso, no es de sorprender que, para este tipo de sucesos, exista un impacto en la vida de las personas, durante los brotes de enfermedades, la ansiedad de la comunidad puede aumentar después de la primera muerte (Rubin, G. y Wessely, S., 2020). La cuarentena implica algo más que la prevención contra la propagación del virus, siempre se la ha utilizado en epidemias por lo que las personas, al escuchar esta palabra y ser privadas de la libertad que tenían antes, por obvias razones afectará la estabilidad psicológica del individuo. Según Rubin, G y Wessely, S. (2020), tenemos cuatro puntos por lo que la cuarentena genera un aumento de la ansiedad en las personas, en primer lugar, esto demuestra que las autoridades ven la situación actual como grave, en segundo lugar, el encierro es más para beneficio de terceros que están fuera de su territorio que para ellos mismos, el tercer punto es que la cuarentena indica una pérdida de control y una sensación de estar atrapados y como último punto el incremento de los rumores y como éste genera alarme

Las epidemias han demostrado tener un gran impacto negativo para la salud psicológica del individuo. Según el Centro para el Control y la Prevención de Enfermedades (CDC) (2020), la pandemia puede resultar estresante para las personas. El temor y la ansiedad con respecto a una nueva enfermedad y lo que podría suceder pueden resultar abrumadores y generar emociones fuertes, tanto en adultos como en niños. Además, se encontró que las medidas de salud pública, como el distanciamiento social, pueden hacer que las personas se sientan aisladas y en soledad y es posible que aumente el estrés y la ansiedad.

En el pasado, con sucesos como las epidemias, se han realizado estudios orientados al impacto psicológicos que estos tienen sobre el ser humano, dentro de esos estudios se realizó uno a causa de un brote por el Síndrome de Respiración de Oriente Medio (MERS) los 
resultados obtenidos fueron con una muestra de 1.656 personas, se realizaron estudios para medir la ansiedad y sentimientos de ira, cuyos porcentajes para ansiedad era de $7,6 \%$ y sentimiento de ira del $16,6 \%$ durante el aislamiento. Luego de cuatro a seis meses de la liberación los porcentajes se encontraron en el $3.0 \%$ para ansiedad y $6.4 \%$ para sentimientos de ira (Jeong, H. Woo, $H$. Song, $Y$. Ki, M. Min, J. Cho, J. y Chae, J., 2016). Con lo que se puede observar la influencia que tuvo la cuarentena en los niveles de ansiedad de la población.

Uno de los resultados de las epidemias, es la cantidad de información que se extiende en la televisión, internet y otros medios de comunicación, gran parte de ella tiene contenido falso que genera alarme en las personas que han obtenido información de carácter desinformativo. Según la OMS (2020) la respuesta que se ha dado en medios de comunicación está acompañados por una infodemia masiva, lo que dificulta que las personas encuentren fuentes confiables y orientación fidedigna cuando es necesaria.
La infodemia es la cantidad masiva de información en un tiempo de estimación muy corta, a causa de un suceso que desencadene este acto (como la pandemia actual). Lo cual, esta epidemia de información contribuye a la desinformación o falsa información, generando estragos en varios aspectos de la vida de las personas, como en el área de la salud mental.

Lo que nos lleva a tres puntos importantes en el impacto que tiene la cuarentena sobre la salud mental en la población: La saturación de información sobre la pandemia, la gran cantidad de personas contagiadas, y el giro que dio la vida cotidiana de las personas (Cuarentena). Todos estos puntos, nos conducen a la repercusión que tuvo este evento, hacia el ser humano y como éste puede generar cambios a niveles psicológicos y físicos. En un estudio de MorenoProaño (2020) citando a Dugas (1995), existe cuatro procesos cognitivos en el mantenimiento de la ansiedad:

1) Intolerancia a la incertidumbre

2) La tendencia a sobreestimar la utilidad de la preocupación 
3) Orientación ineficaz frente a los problemas y

4) la evitación cognitiva.

Por lo que podemos encontrar concordancia con los tres puntos expuestos anteriormente, y el aumento en los niveles de ansiedad del individuo

Como se mencionó anteriormente, la respuesta de las personas ante tal evento, a nivel mental es el incremento de lo que se denomina "ansiedad". Estudios han encontrado ciertos hallazgos que son consistentes con los estudios sobre el SARS, en Taiwán donde hasta el $75 \%$ de los trabajadores de la salud experimentaron morbilidad psiquiátrica. Además, se encontró en Singapur 238 casos de SARS, con una morbilidad psiquiátrica de hasta el 21\%. (Sim y Choon, 2004; Dr. Mian-Yoon Chong, Chang Gung Memorial Hospital, Kaohsiung, 2004)

Es importante saber qué es la ansiedad, como funciona y por qué está presente en la vida de las personas. A lo largo del tiempo, se le ha dado muchas definiciones. Según Spielberg (1980): "Es una reacción emocional desagradable producida por un estímulo externo, que es considerado por el individuo como amenazador, produciendo ello cambios fisiológicos y conductuales en el sujeto". Beck (1985): "Es la percepción del individuo a nivel incorrecto, estando basada en falsas premisas.". Lang (2002): "Es una respuesta emocional que da el sujeto ante situaciones que percibe 0 interpreta como amenazas 0 peligros.".

Teniendo en cuenta los diferentes conceptos expuestos, podemos decir que la ansiedad es una respuesta a una situación, objeto o cosa que genere incertidumbre 0 malestar hacia la persona. La presencia de ansiedad en la vida de las personas es algo que no se puede negar, el problema radica en el aumento de los niveles de esta, y con ello, que se vea afectada la vida del ser humano (salud mental y física). Según Lorenzo-Ruiz, A., Díaz, K., \& Zaldívar, D. (2020), al ser tan compleja y variable esta situación a nivel mundial, es posible integrar el conjunto de estas manifestaciones clínicas psicológicas, incluyendo manifestaciones psicofisiológicas tales como insomnio, pesadillas, disminución o pérdida del apetito, abandono de hábitos higiénicos, 
entre otros. Lo que nos lleva a observar cómo puede ser afectada la calidad de vida del ser humano.

Cuando ocurre un cambio significativo en la vida de las personas y más aún, que nos coloque en un estado de incertidumbre que van contra el bienestar del individuo, se activan ciertos mecanismos de defensa en contra de la amenaza que atente contra la salud de la persona, dicho de otra forma, los procesos cognitivos del individuo comienzan a trabajar para evitar algún tipo de daño, a esto se lo conoce como funciones o estrategias adaptativas (Aron T. Beck y Emily AP. Haigh., 2014). Estas funciones nos permiten obtener bienes físico y mental. Pero dichas funciones, dejan de ser funcionales y pasan a ser disfuncionales cuando una interpretación es errónea y exagera a una menaza y da como resultado ansiedad. Es así como en conjunto, todos estos factores pueden generar que la mente suba sus defensas y exista un aumento en los niveles de ansiedad.

Es por todo esto, que es de suma importancia caracterizar la influencia de la cuarentena en los niveles de ansiedad de los ciudadanos de la Parroquia Ángel Pedro Giler del Cantón Tosagua

\section{Metodología (Materiales y métodos)}

Se desarrollará un estudio descriptivo con un enfoque cualitativo-cuantitativo cuyo propósito es describir situaciones y eventos cual manifiesta determinado fenómeno.

El método que se escogió fue el Inductivo-deductivo puesto que la inducción es un modo de razonar que consiste en sacar de los hechos particulares una conclusión general, es un razonamiento que analiza una porción de un todo, por lo que va de lo particular a lo general. La deducción es un método de razonamiento que lleva a conclusiones partiendo de lo general, aceptado como válido, hacia aplicaciones particulares.

Entre las técnicas a aplicar se consideran la observación, y la aplicación de test de escala de ansiedad Beck, un cuestionario de opción múltiple de 21 ítems que mide la gravedad de la ansiedad actual en adultos y adolescentes. Describe los 
síntomas emocionales, fisiológicos y cognitivos de la ansiedad, siendo una prueba muy adecuada para discriminar la ansiedad de la depresión. Y el test de DASS-21 que es una escala para medir la depresión, ansiedad y estrés, cuenta con 21 ítems, con cuatro alternativas de respuesta en formato Likert, las cuales van desde 0 ("No describe nada de lo que me pasó o sentí en la semana") hasta 4 ("Sí, esto me pasó mucho, o casi siempre").

Las técnicas aplicadas se llevaron a cabo en la población de la parroquia "Ángel Pedro Giler", con una población de 6280 habitantes, se escogió una muestra de 25 personas correspondiente a un rango de edad de 18-35 años sin distinción de sexo determinando el tipo de muestreo de carácter intencional.

Se contactó a la población correspondiente a la muestra establecida por medio de redes sociales como Facebook, Instagram y WhatsApp, una vez establecida la conexión, se procedió a la aplicación de un cuestionario y dos test (ansiedad de Beck y Dass-21), se realizó de manera virtual, utilizando la plataforma de Google Forms para llevar a cabo la recolección de los datos, una vez calificada de manera individual los resultados, se empleó estadística descriptiva a través de la prueba de Kruskal-Wallis para la obtención de información.

\section{Resultados}

Los datos estadísticos que se obtuvieron fueron realizados por prueba de Kruskal-Wallis, dentro de los cuales se obtuvieron datos significantes para la presente investigación. Se uso una muestra, aplicando el cuestionario de Dass-21 y el inventario de Beck de ansiedad. Obteniendo resultados con valores menor ( $p$ valor <0.05). en el cuestionario de Das.

Cuando las personas perciben alguna molestia en su cuerpo, ¿piensa que podría estar infectado/a por el virus Covid-19? causó ansiedad y estrés, y depresión a un nivel significante $\quad(p<0.05)$. Asimismo, en la pregunta "En su familia es un tema habitual la pandemia". en La prueba de Kruskal-Wallis indica que los resultados para las escalas de Dass21: Depresión, ansiedad y estrés son estadísticamente significante (p valor $>0.05)$. 
En el gráfico de cajas y bigotes para los resultados Dass-21 hay un mayor índice de ansiedad para las personas que se informan sobre la pandemia solo por medio del internet, en comparación con las que se informan de otros medios de comunicación. Lo cual también los resultados de este estudio corroboran que las personas que reciben información del internet sufren más ansiedad. Muchas de las preguntas tenían una mayor distribución en el gráfico de cajas y bigotes para los resultados de Dass21 y Beck, lo cual puede ser indicación de lo severo que ha sido la pandemia.

Los datos estadísticos obtenidos, fueron realizados por la prueba de Kruskal-Wallis, dentro de los cuales se obtuvieron datos significantes para la presente investigación. Teniendo como nivel de significancia valores menor (p. <0.05). Como también En el gráfico de cajas y bigotes se puede observar que muchas preguntas presentaron mayores valores con mayores frecuencias

Se aplicó el cuestionario Dass-21 y el inventario de Beck de ansiedad. Las personas que contestaron " $\mathrm{Si}$ perciben alguna molestia en su cuerpo, piensan que podrían estar infectado/a con el virus Covid-19". Alcanzaron resultados que demostraron un mayor índice en el cuestionario de Dass-21: en todas las subescalas ansiedad, estrés y depresión, esta categoría si influye estadísticamente en estos parámetros con valores menores ( $p$. $<0.05)$. Las personas que respondieron que "Si" en el ítem "Si percibe alguna molestia en su cuerpo, ¿piensa que podría estar infectado/a por el virus Covid-19?", presentan una mayor distribución con mayores valores, en el cuestionario de Dass-21: en ansiedad, estrés y depresión, y se pronosticaría que las personas que si pensarían estar contagiados presentarían valores más altos en todas las subescalas del cuestionario de Dass-21.

De la misma forma, en el gráfico de cajas y bigotes para los resultados de Beck se observa una mayor distribución para las personas que marcaron "Si" en el ítem "Si perciben alguna molestia en su cuerpo, piensan que podrían estar infectado/a con el virus Covid-19", con mayores valores, por lo cual, a 
pesar de no tener diferencia estadísticamente para esta categoría se deduce que estas personas presentan mayores resultados en Beck con mayores valores y frecuencias. En siguiente gráfico se obseva que en el cuestionario de Dass se observo de que las personas que trabajan tienen mayores ansiedad.

Figura 1. Prueba Kruskai-Wallis muestras independientes pregunta 5.

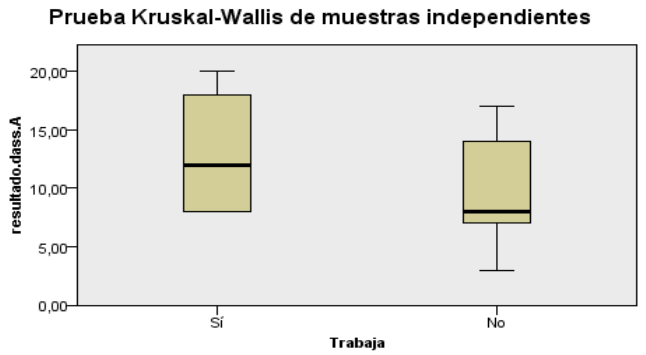

Fuente: Veléz (2020)

La figura 1. Indica que hay una mayor distribución, para las personas que si trabajan en relación de la que no trabajan con mayores valores de ansiedad

En el gráfico de cajas y bigotes se puede observar que la subescala de ansiedad en el cuestionario Dass21 se observa una mayor distribución para las personas que si trabajan con mayores valores, por lo que a pesar de que estadísticamente no presentar diferencias, mediante el gráfico se deduce que las personas que trabajan presentan los mayores valores con mayores frecuencias en subescala de ansiedad

Del mismo modo existe un rango más amplio de distribuciones superiores para los que trabajan por lo que a pesar de estadísticamente no presentar diferencias, mediante el gráfico se deduce que las personas que trabajan presentan los mayores valores con mayor frecuencia en la subescala de estrés y depresión de Dass-21 hay una mayor distribución, para las personas que si trabajan en relación de la que no trabajan siguiendo el mismo patrón de subescala de ansiedad.

En el ítem dirigido hacia los medios de comunicación sobre la pandemia se puede considerar que internet fue el medio que aumento el efecto de ansiedad.

Figura 2. Prueba Kruskai-Wallis muestras independientes pregunta 11.

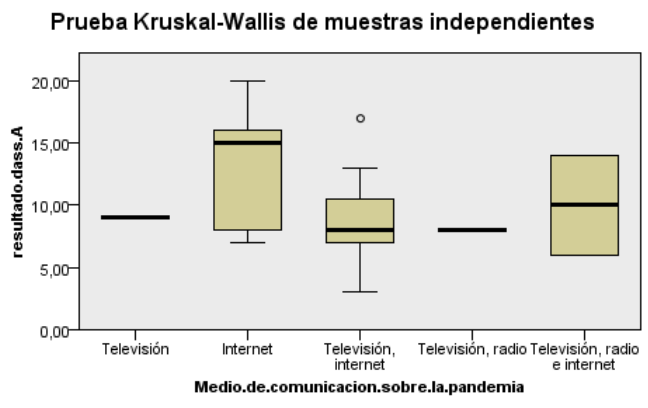

Fuente: Veléz (2020). 
La figura 2. Se observa que hay una mayor distribución, para las personas que se informan sobre la pandemia solo por medio del internet, en comparación con las que se informan de otros medios de comunicación

Pese a que no hay diferencias para esta categoría, se puede observar en el gráfico de cajas y bigotes para los resultados de Dass-21 hay un índice mayor de ansiedad para las personas que se informan sobre la pandemia solo por medio del internet, en comparación con las que se informan de otros medios de comunicación.

Con respecto al caso del internet, la Distribución es asimétrica negativa porque la mediana está más cerca del tercer cuartil Q3 (cuartil superior). A diferencia del caso anterior, la distribución de la variable televisión, radio e internet es Simétrica con relación a la mediana, distribuyéndose de forma pareja las respuestas entre los encuestados, esto demuestra además que la mayoría de las personas utilizaron estos medios de forma combinada para informarse.

Al analizarlos de forma individual, televisión y radio no tienen respuestas afirmativas entre los encuestados, lo que hace notar que el internet es un medio que tiene mucho más uso y acogida en la actualidad para informarse sobre la situación.

Figura 3. Prueba Kruskai-Wallis muestras independientes pregunta 12.

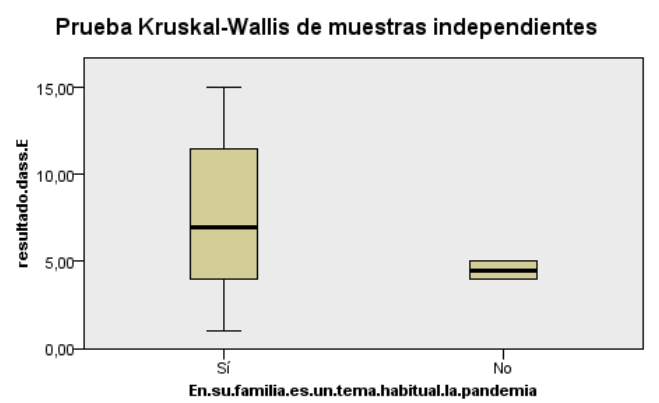

Fuente: Veléz (2020)

La figura 3. Muestra el gráfico de cajas y bigotes para los resultados de Dass-21 estrés se observa una mayor distribución para las personas que si hablan sobre la pandemia con mayores valores.

También se apreció que en inventario de Beck de ansiedad que influye en cuando contestaron "si" y "poco" versus "no" a la pregunta "en su familia es un tema habitual la pandemia" surgió ser estadizamente significante cuando se unieron las respuestas si y poco con un nivel de significancia de ( $p \_$valor $\left.>0.024\right)$.

En el gráfico de cajas y bigotes para los resultados de Dass-21 estrés se 
observa una mayor distribución para las personas que si hablan sobre la pandemia un mayor grado de estrés, por lo que, a pesar de estadísticamente no presentar diferencias, mediante el gráfico se deduce que estas personas presentan los mayores valores con mayor frecuencia para los resultados de Dass-21 estrés a diferencia de las que no hablan sobre este tema. Con la misma tendencia para las subescalas de Dass-21 de depresión y ansiedad.

Figura 4. Prueba Kruskai-Wallis muestras independientes pregunta 14.

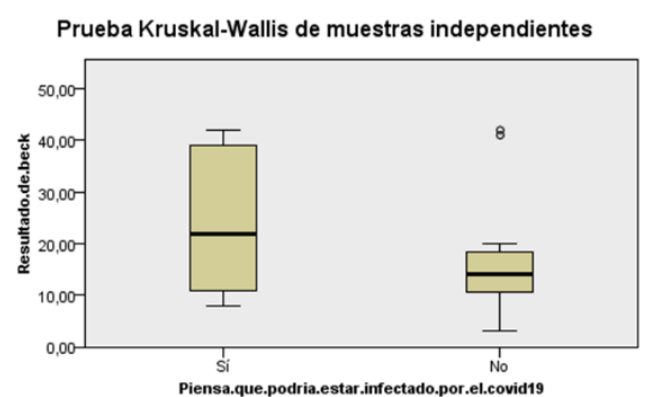

Fuente: Veléz (2020).

En el gráfico de cajas y bigotes para los resultados de Beck se observa una mayor distribución para las personas que percibiendo alguna molestia en su cuerpo, pensarían que podrían estar infectado con el virus Covid-19 con mayores valores, por cual a pesar de no tener diferencia estadísticamente para esta categoría se deduce que estas personas presentan mayores resultados de Beck con mayores frecuencias.

Al observar el grafico correspondiente a las personas que consideran que podrían estar infectados por el covid19, la distribución de los datos genera una representación asimétrica positiva encontrándose la mediana más cerca del primer cuartil Q1 (cuartil inferior), lo que indica que la media es mayor que la mediana y mayor que la moda.

En referencia a las respuestas de los encuestados, la mayoría de ellos presenta un cuadro de ansiedad que les provoca la sensación de presentar alguno de los síntomas característicos de la enfermedad por covid19, en la mayoría de los casos, sin haber estado expuestos a la misma, lo cual ha sido propiciado principalmente por las noticias observadas en los diferentes medios de comunicación.

En el otro extremo, se encuentran aquellas personas que, en un menor número, no han tenido la sensación de estar contagiados ni han experimentado los síntomas de la enfermedad, los cuales en la representación permiten observar 
datos más dispersos y con menor variabilidad expresada en las respuestas.

Figura 5. Prueba Kruskai-Wallis muestras independientes pregunta 15.

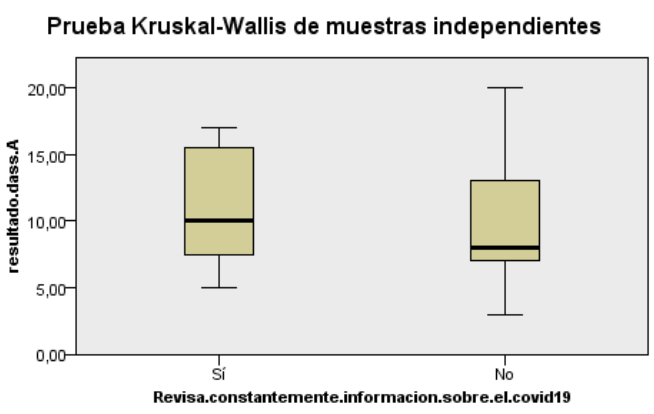

Fuente: Veléz (2020).

En el gráfico de cajas y bigotes para los resultados de Dass-21 en ansiedad, se observa una mayor distribución para las personas que revisan constantemente información sobre el covid19, sin embargo, los límites de los que no revisan constantemente información son mayores por cual no se tiene diferencia estadística ni gráfica para esta categoría.

Con respecto a la interrogante sobre la posibilidad de revisar constantemente la información relacionada al covid19, se puede observar que la gran mayoría de los encuestados afirman que es una situación habitual, debido a la gran cantidad de información, muchas veces no comprobada, que se encuentra disponibles en diferentes medios, principalmente en el internet y las redes sociales, en este caso el gráfico de caja y bigotes nos muestra una distribución asimétrica de los datos, teniendo una media mayor que la mediana y mayor que la moda.

Finalmente, los datos sobre aquellas personas que no consumen información sobre el covid19 se encuentran muy dispersos y con poca variabilidad, demostrando que, en comparación con aquellos que respondieron afirmativamente a la interrogante, este fue un grupo mucho más reducido de personas.

\section{Conclusiones}

Es bastante evidente que la pandemia del covid-19 ha causado mucha ansiedad, estrés y depresión en muchas personas. Como el Centros para el Control y la Prevención de Enfermedades (CDC, 2020) que considera que esta pandemia puede ser muy estresante. Muchos de los resultados de este trabajo muestran que pandemia ha afectado nuestro estado emocional como ansiedad y depresión. Quizá, no hemos estado muy conscientes del grado de afectación que ha 
tenido la pandemia sobre la salud mental de las personas, principalmente en los niveles de ansiedad y estrés, ocasionando un desgate psicológico, teniendo como referencia el ítem "Si percibe alguna molestia en su cuerpo, ¿piensa que podría estar infectado/a por el virus Covid-19?". Ya que la misma ansiedad nos provoca ese sentimiento de estar infectado. Es importante señalar que la ansiedad está relacionada con el miedo de perder la vida o sea una ansiedad de sobrevivencia.

En relación con los resultados de la pregunta "en su familia es un tema habitual la pandemia" el mismo miedo o temor hace que en la familia, tomen el tema de la pandemia. Entrando de esta manera, en un círculo vicioso que da como resultado el aumento de ansiedad en torno al tema especificado anteriormente. Los resultados son: las personas que hablan sobre el tema de la pandemia sufren un aumento en sus niveles de ansiedad, estrés y depresión.

Sabemos que los medios de comunicación tienen un efecto potencial sobre las personas por eso es importante que las personas puedan discernir la información ya que los gobiernos poseen poco 0 ningún control sobre las redes sociales, las personas reciben una cantidad excesiva de información. Muchos estudios demuestran que el COVID 19 ha estado acompañado con una gran cantidad de información que no ayuda a las personas encontrar información que les ayude entender la situación OMS (2020). Lo cual también los resultados de este estudio corroboran que las personas que reciben información del internet sufren más ansiedad, estrés y depresión.

Muchas de las preguntas tenían una mayor distribución en el gráfico de cajas y bigotes para los resultados de Dass-21 y Beck lo cual puede ser indicación de lo severo que ha sido la pandemia en la psique del individuo. También hay que tomar en cuenta que la muestra no sido tan grande para sí tener mejores resultados sin embargo se puede percibir el efecto de la pandemia en nuestro estado emocional

Los resultados del estudio permiten proponer estrategia para una intervención más efectiva puesto que podemos observar, por ejemplo, el 
efecto que tiene cuando uno siente síntomas psicológicos del COVID 19 causando depresión, ansiedad, y estrés de que hayamos pensado. Como también el efecto que tiene los medios de comunicación en nuestro estado emocional, especialmente el internet. Esto resultado nos pueden ayudar para educar a la población que sepan discernir la información así podría reducir la ansiedad, el estrés y la depresión

La psicoeducación sobre el virus Covid-19 debe ser constante, puesto que el temor de las personas por la pandemia actual, en compañía a la desinformación que muchos sitios de internet proporcionan da como resultado la afectación del bienestar psicológico de las personas. Como también hacer consiente a la población de que estar continuamente hablando con la familia sobre la pandemia incrementa la ansiedad, esto podría llevarse, de igual manera a través de intervenciones psicoeducativa o en las intervenciones de crises que el terapeuta este consiente, de esto factores.

\section{Bibliografía}

Centros para el control y a prevención de enfermedades. (2020). Enfermedad del coronavirus 2019. Extraído de:

https://espanol.cdc.gov/coron avirus/2019-ncov/daily-lifecoping/managing-stressanxiety.html

Jeong, H. Woo, H. Song, Y. Ki, M. Min, J. Cho, J. y Chae, J. (2016). Mental health status of people isolated due to Middle East Respiratory Syndrome. Extraído de: https://doi.org/10.4178/epih.e 2016048

Oblitas, A. y Sempertegui, N. (2020). Ansiedad en tiempos de aislamiento social por COVID19. Chota, Perú - 2020. Extraído de: https://revistas.unal.e https://publicaciones.ucuenca .edu.ec/ojs/index.php/medicin a/article/view/3224du.co/inde x.php/avenferm/article/view/8 7589

Organización Panamericana de la Salud. (2020) ¿Qué es la infodemia?

Organización Panamericana de la Salud. (2020). Enfermedad por el Coronavirus (COVID19). Extraído de https://www.paho.org/es/tag/e nfermedad-por-coronaviruscovid-19 
Organización mundial de la salud. (2003). El brote de SRAS ha sido contenido en todo el mundo. Extraído de: https://www.who.int/mediacen tre/news/releases/2003/pr56/ es/

Ortiz-Muñoz, L. E., Pantoja, T., \& Morel, M. (2020). Resumen SUPPORT de una revisión sistemática: Cuarentena sola o en combinación con otras medidas de salud pública para controlar el COVID-19: una revisión rápida. Satélite Cochrane EPOC-CHILE. Extraído de: https://osf.io/preprints/kx6pn/

Rodríguez, $\quad \mathrm{T}$. Fonseca, $\mathrm{M}$. Valladares, A. y López, L. (2020). Protocolo de actuación psicológica ante la COVID-19 en centros asistenciales. Cienfuegos. Cuba. Extraído de: http://scielo.sld.cu/scielo.php? pid=S1727-

897X2020000300368\&script= sci_arttext\&tlng=pt

Lorenzo, A. Díaz, K. y Zaldívar, D. (2020) La Psicología como Ciencia y Profesión en el Afrontamiento del COVID-19. Extraído de: https://revistacaribenadepsico logia.com/index.php/rcp/articl e/view/4815

López, L. Valladares, A. y Canel, O. (2020). Propuesta metodológica para el abordaje psicológico de los prestadores de salud a las personas en centros de aislamiento por COVID 19. Extraído de: http://scielo.sld.cu/scielo.php? pid $=$ S1727-

897X2020000300352\&script= sci_arttext\&tlng=en

Moreno-Proaño,

G.

(2020).

Pensamientos distorsionados y ansiedad generalizada en COVID-19. Extraído el 15 de septiembre del 2020 de: http://cienciamerica.uti.edu.ec /openjournal/index.php/uti/arti cle/view/314

Ministerio de Salud Pública. (2020). El MSP informa: Situación coronavirus Covid-19. Extraído de: https://www.salud.gob.ec/elministerio-de-salud-publicadel-ecuador-msp-informasituacion-coronavirus/

Ministerio de Salud Pública. (2020). Organización comunitaria y nuevos hábitos serán clave para la fase de distanciamiento social. Extraído de: https://www.salud.gob.ec/org anizacion-comunitaria-ynuevos-habitos-seran-clavepara-la-fase-dedistanciamiento-social/

Ministerio de Salud Pública. (2020). Comportamiento CoVID-19 (PCR) Ecuador. Extraído de: https://www.coronavirusecua dor.com/data/ 
Ministerio de Salud Pública. (2020).

Preguntas frecuentes sobre salud mental. Extraído de: https://www.coronavirusecua dor.com/conoce-tu-saludmental/\#1598054738679bf800cfc-c1dc

Rubin, G. y Wessely, S. (2020). The psychological effects of quarantining a city. Extraído de:

https://www.bmj.com/content/ 368/bmj.m313.full

Rodríguez, $\quad \mathrm{T}$. Fonseca, $\mathrm{M}$. Valladares, A. López M. (2020). Protocolo de actuación psicológica ante la COVID-19 en centros. Extraído de: http://scielo.sld.cu/pdf/ms/v18 n3/1727-897X-ms-18-03368.pdf

Sim, K. y Choon, H. (2004). The psychological impact of SARS: a matter of heart and mind. Extraído de: https://www.cmaj.ca/content/1 $70 / 5 / 811$ 